\title{
TUMOURS OF THE BLADDER.
}

\author{
By R. OGIER WARD, M.Ch. (Oxon.), F.R.C.S. \\ (Assistant Surgeon, St. Peter's Hospital for Stone.)
}

There is probably no branch of urology more specialized than that which deals with the diagnosis and treatment of bladder growths for both are dependent in large measure upon cystoscopic procedures. Their existence can only be proved with certainty by this method, their nature can usually be determined by the appearances when so examined, and the treatment of the condition is in a large proportion of cases best undertaken by transurethral operations.

\section{PAPILLOMA.}

Itiology. The cause of this condition is no more understood than that of any other new growth. Papillomata are more commonly found in the neighbourhood of the ureters, often so near to them that the opening is obscured, being frequently placed just above it. In other instances they occur on the sides and floor of the bladder adjacent to this region. The development of the bladder is complex in this part, which may perhaps explain why this area is the region most commonly affected. Papillomata are uncommon anywhere on the trigone, though recurrences are not infrequently seen at the internal urinary meatus.

The only known predisposing cause exists in the case of workers in aniline dyes who are specially susceptible to this disease; apparently it is some substance excreted in the urine.

It is not known how long a papilloma takes to develop because, until hæmaturia occurs, there is usually no indication of its existence. In one patient, a male, aged 48, who had suffered from renal colic, cystoscopy showed the bladder normal except for a calculus protruding from the ureteric orifice; five years later the onset of hæmaturia led to this examination being repeated, when a simple papilloma of considerable size was now found obscuring the opposite duct. It is, however, often observed that recurrences after treatment develop in a few months, and those which are malignant do so with special rapidity.

Symptoms. The typical symptom of vesical papillomata is hæmaturia alone, occurring unexpectedly at intervals of months or longer, but tending to become more frequent as the growth increases. The blood is sufficiently abundant to be very obvious to the patient; it may continue for a few days or only be present on one or two occasions. Papillomata are occasionally found by chance which have not produced even this symptom, but I have not seen an example of this which was larger than a cherry, though many growths are much bigger than this by the time the patient first comes for examination.

The larger growths sometimes give rise to difficulty in micturition particularly when they are near the bladder outlet and may prevent its proper emptying. A female patient, aged 68, had suffered from hæmaturia eight years previously, then 
again after a lapse of six years and next about nine months before investigation. Recently the papilloma, which was a large one, had occasionally prolapsed through the urethra into the vulva to such an extent that the pedicle could be palpated. This had not produced retention of urine, but there had been much pain and frequency although the urine was not infected.

Diagnosis. On cystoscopy some villous papillomata can often be confidently recognized as being benign. The characteristics of such growths are that they are single, that they are pedunculated, and that they have a very delicate structure, the fronds being long, slender and translucent, a single fine vessel running along each of them. These features can be seen with any cystoscope, but are specially well studied if a single catheterizing, flushing instrument be used. The mobility of the growth, on the bladder wall and of its constituent villi, are then made very obvious when fluid is allowed to flow over it, and if a diathermy electrode be passed the growth can often be pushed aside sufficiently to expose the pedicle, this being perhaps more easily done after some part has been treated by coagulation. Other papillomata can be as certainly recognized as malignant in nature. These are of much more solid appearance, the villi being short or absent; they are more vascular; there is no pedicle; odema surrounds the base and small nodules of growth show in the mucosa close by. The urine is septic. But between these two extremes there occur many varieties of papilloma presenting difficulties in diagnosis. There are a few facts worth remembering in this connection. The younger the patient the more likely is the growth to be innocent; the converse, however, does not hold. Growths which are pedunculated are probably benign, those which are sessile are more probably malignant. If the villi are stunted or absent, when they are often called bald papillomata, there is a strong chance of malignancy. Sepsis shown by urinary infection, degeneration of parts of the growth or a covering of phosphates, is strong evidence of a carcinomatous nature. A tumour, the outline of which can be palpated, is certainly malignant.

Growths which are single must not for that reason alone be considered innocent, unless they conform to the description already given, and multiplicity does not imply malignancy, though multiple recurrences occurring after a papilloma has been excised are usually of this nature. Extensions around the main growth, like velvet pile, do not indicate malignancy even though of considerable spread, but the presence of œedema always means submucous infiltration and therefore cancer.

Fragments of papillomata can often be obtained for microscopy during endoscopic treatment, and of course if the operation is an open one, but the results thus obtained are of little value, for it is only when the bladder wall is infiltrated that the microscope can provide a diagnosis which is final. The opinion arrived at during cystoscopy is the most important part in diagnosis; second to this is that obtained from bimanual examination, an investigation which should never be omitted. A simple papilloma cannot be palpated unless it is unusually large and is then only felt as an elastic swelling, not well defined. A malignant papilloma when there is submucous invasion can not infrequently be felt in the female, but this is more difficult in the male. In a thin female who had five growths in the 
bladder I was able, from bimanual vaginal examination, to distinguish one papilloma which in diameter was less than a centimetre. This was a malignant bald papilloma, the others were villous.

Treatment. Papillomata should never be left untouched as they may be expected to increase in size; hæmaturia will become more profuse and sepsis may result. Every case which seems likely to respond to endoscopic treatment should be given a trial of this method in the first instance. Small growths respond well to perurethral diathermy, it is only the largest which require excision and this always carries with it a risk of implantation of the growth in the scar; such recurrences are malignant and often spread widely outside the bladder towards the abdominal wall.

The smallest growths, and small recurrences after diathermy, can be treated without anæsthesia, but in the case of medium and larger growths, I prefer always that the patient be anæsthetized, a low spinal injection of percaine with preliminary administration of morphia and scopolamine is particularly suited for this purpose, there being no need to obtain anæsthesia of the abdominal wall. Under these conditions the treatment can be prolonged if the growth is large or if there are several of them. Smaller growths can usually be entirely destroyed together with the immediately surrounding mucosa which is impossible without anæsthesia, and this seems to lessen the chances of recurrence. The velvet-like extensions of papillomata or the underlying mucosa are often very sensitive and cannot be cauterized without anæsthesia. This at the same time permits of repeated washing of the bladder which is necessary for good visibility when there is much to be done. It may be added that the use of a flushing cystoscope helps greatly to retain a clear medium. The usual type of diathermy electrode is suitable for the smallest growths, but for all others I prefer one ending in a ball of the maximum size which can be passed through the sheath of one of the special cystoscopes necessary for this work. Much more destruction can be effected in this manner and in a much shorter time. If care is taken to maintain visibility and not to overdistend the bladder, the only risk is that of producing obstruction to a ureter. This may result, if too intensive diathermy is used on a growth situated close to the duct, and if this occurs it may be serious as it lasts for some days. Diathermy treatment may be tried for every type of papilloma, except those which give signs of extension beneath the mucosa, the evidence of this being cedema with or without surrounding nodules, and a defined solidity which can be recognized on bimanual examination. Except in the case of bald papillomata this latter means that the growth is no longer only of the mucosa, and that cystoscopic diathermy alone is therefore useless. Open operation then becomes the best form of treatment; the methods will be discussed with the treatment of other malignant growths.

Every case of vesical papilloma which has been treated should be cystoscoped after an interval. If the first diathermy has been incomplete, and this is usually so if the growths are large or numerous, this can be done after three weeks. In every case the examination should be repeated at intervals of six months and for a period of two or three years after an apparent cure has resulted. If a solitary papilloma proves too large to be destroyed in a few cystoscopic treatments, then the bladder should be opened and the growth coagulated in situ with the minimum 
of disturbance; the base can then be excised. If a peduncle is found which can be cut through and the growth thus removed complete, this is the best treatment. In the case of multiple growths the cystoscopic method of treatment should be given extended trial as it often suffices.

\section{MALIGNANT GROWTHS.}

Malignant papillomata have already been described. They may be primary or they may occur as recurrences after open excision of apparently innocent growths, and it is said that innocent growths may change their character in this direction. Other forms of cancer are nodular infiltrating growths, squamous-celled carcinomatous ulcers, and sarcoma, the latter being very rare. The sites in the bladder affected by these growths are the same as those for papillomata, but they extend more frequently on to the trigone and to the internal meatus than do the latter.

Symptoms and Signs. Hæmaturia always occurs at some stage, but not infrequently when the patient is first seen the growth is found to be a large one though bleeding has only recently begun. Hæmaturia is at shorter intervals than in simple growths and tends to become more profuse and with clots. The pain of cystitis is sometimes the first symptom. Cystitis always occurs sooner or later, and with the concomitant frequent micturition induces an extreme degree of suffering. The urine is seen to be bloodstained and septic and to contain considerable debris from the necrotic growth and the inflamed bladder wall. The quantities passed are small and the patient presently looks ill from loss of sleep and pain. Sepsis and the general effects of malignant disease contribute to this.

Diagnosis. Cystoscopic Examination. This is clearly the most important method of diagnosis but often may be too painful to be carried out unless under anæsthesia. It should never be postponed merely on account of hæmaturia, for even if this is considerable, provided a flushing cystoscope be employed, it is almost always possible to get a view sufficient to recognize the presence of a growth. The appearances of malignant papillomata have already been described. Of the two other varieties the infiltrating carcinoma is much the commoner. This takes the form of one or more red raised nodules of solid growth, their surface often being necrotic and sloughing, œdema exists round the bases and an intense local congestion is associated with generalized cystitis. The bladder capacity is diminished and the growths bleed readily especially if the bladder is vigorously irrigated. Epithelioma is a much less common form; such growths have an ulcerated base and a raised indurated edge.

A growth arising just above the internal meatus anteriorly may be missed, for in the male this part of the bladder wall cannot be seen with the ordinary rightangled view cystoscope. An example of this was a man aged 50 who gave a history of renal colic during the previous two years with slight hæmaturia recently. When cystoscoped a calculus was seen protruding from the right ureteric orifice; this was cauterized through the cystoscope and the stone was passed after five days. The bladder was otherwise healthy and the urine was clear. Two years later he reported that his bladder was irritable and that there were now small particles 
of blood in the urine, but that he had had one considerable hæmaturia twelve months ago. Cystoscopy was once more carried out but as this showed only a normal bladder (there was no hæmaturia at the time) it was arranged that this should be repeated during the next attack of hæmorrhage. A slight enlargement of the prostate was recognized and thought to be a possible source of the bleeding. Eleven months afterwards he reported for further investigation as he had been bleeding considerably for a period of two months. His frequency of micturition had increased and it was painful. He had lost weight and he looked very ill. Bimanually a large mass could be felt between the rectal and abdominal fingers. Cystoscopy now revealed the projecting surface of a huge carcinoma which had clearly grown from the bladder wall above the internal meatus. It was obvious that the prevesical space was infiltrated and that the peritoneum was involved. $\mathrm{He}$ died three months later, being for the last two quite free of hæmaturia. A remarkable feature of this case was that the urine was never offensive and remained sterile on culture to the last though a small amount of pus was present. One benefit resulted, for when his brother aged 60 developed hæmaturia after coitus, he reported the fact at once and when cystoscoped was found to have a bald papilloma half an inch in diameter at the apex of the bladder. This could just be palpated bimanually. It was resected with a wide margin of healthy bladder wall and a portion of peritoneum. The prognosis in this patient may be considered favourable.

The second and most important manner of making a diagnosis and in deciding the suitable treatment is bimanual examination. Unless the patient is obese or unable to relax the abdomen, it will be possible in a large number of cases to recognize by this method that a bladder growth is malignant, and the proportion will be increased if this is done whilst the patient is under an anæsthetic. By the time the patient comes for examination a nodular infiltrating growth commonly has an extent in and outside the bladder wall which is much greater than that on the surface of the mucosa. Indeed, a growth which seems small on cystoscopy often already forms a considerable tumour. It should be noted that the coincidence of an enlarged prostate will make it impossible to obtain the desired information by this means.

Treatment. Excision is the best treatment for every type of malignant bladder growth and should be carried out if there is the least prospect of success, for the reason that these conditions lead to intense suffering if nothing can be done to cure them. Growths arising on the anterior surface of the bladder are fortunately uncommon as they are the least favourable, for so often they are found to be adherent to the abdominal wall. Those situated in the neighbourhood of the air bubble which is seen on cystoscopy, or below this region, will often require that the peritoneal cavity be opened and a portion of that membrane excised. The mobility of such growths and their relation to the abdominal wall can often be accurately estimated by pressure upon the latter during the cystoscopic examination and the same process often helps to bring them into better view. Growths of the base of the bladder and lateral walls frequently cannot be removed without resection of the lower portion of the ureter. The duct is usually dilated above a malignant growth and excretion urography will often show that the function of the kidney is thereby impaired, but this is no contraindication to transplantation of the duct. Growths extending down to the trigone and internal meatus are often met with, 
and to remove them a portion of the prostate may have to be excised. It is desirable to remove a margin of an inch of healthy bladder wall all round the growth, often, however, one has to be content with less than this and the results are not necessarily unfavourable. The whole thickness of the bladder wall should always be excised. It is useless to attempt a block dissection to include glands and lymphatic channels, and fortunately the glands are usually involved only late in the disease. The guiding principle when operating should be to remove from the bladder itself what may be considered to be an intensely irritating foreign body wherever this is possible, death from metastases (which are most commonly found in the pelvic bones and pelvic tissues) being preferable to the suffering of a progressive cystitis. It should be remembered that blood transfusion will often help to make an operation a success. Malignant papillomata should be coagulated in situ before the base is excised.

Deep X-ray therapy has so far proved unavailing in the treatment of bladder growths, innocent or malignant, and radium applied direct to the growth after making a cystotomy incision has not been followed by much success. There is, however, a real place in treatment for radon seeds implanted by cystoscopic methods. This method may be used for malignant papillomata. It is not suited for nodular growths since only one aspect of the tumour can be treated. I have, however, in this way successfully treated a carcinoma arising in a cystotomy scar. In this case seeds were implanted into the nodular mass seen through the cystoscope, and after this through the abdominal wall down into the tumour in the prevesical space, some of them being pushed close down to the bladder mucosa whilst this was being kept under cystoscopic observation. Seeds so used are left in situ, and subsequently are either passed in the urine or remain embedded in the bladder wall. In only two cases in a series of 63 did these give rise to trouble; of these one was a female and two seeds were later found within a phosphatic calculus. This patient, however, had been forming bladder stones at intervals for many months previously. The other case was a male, and a calculus containing one seed was passed naturally some months after treatment. Total cystectomy after the ureters have been transplanted on to the skin or into the bowel, can sometimes be performed but the mortality is high as such patients' powers of recovery are low. This operation is best suited to extensive malignant papillomata confined within the limits of the bladder cavity and its wall.

In conclusion, it may be stated that successful treatment can only be attained in bladder growths if they are seen early, and for this it must be more generally realized that every case of hæmaturia demands the earliest possible investigation. 